\title{
CONDITIONAL ASYMPTOTIC FORMULAE FOR A CLASS OF ARITHMETIC FUNCTIONS
}

\author{
WERNER GEORG NOWAK AND MICHAEL SCHMEIER
}

(Communicated by Larry J. Goldstein)

\begin{abstract}
Under the assumption of Riemann's Hypothesis, a general asymptotic formula for sums $\sum_{n \leq x} a(n)$ is established which applies (e.g.) to arithmetic functions $a(n)$ defined by a kind of convolution of the Möbius function with some divisor function.
\end{abstract}

1. Introduction. In a recent paper [5], H. L. Montgomery and R. C. Vaughan have investigated the distribution of $k$-free integers under the assumption of the Riemann Hypothesis (henceforth quoted as $R H$ ). By a simple but ingenious new idea they succeeded in improving the classical error term $O\left(x^{2 /(2 k+1)+\varepsilon}\right)$ (due to A. Axer [1]) in the formula for the number of $k$-free integers $\leq x$ to $O\left(x^{1 /(k+1)+\varepsilon}\right)$.

The objective of this note is to exploit this idea in a more general context, showing that it admits applications to a large class of arithmetic functions $a(n)$ which are defined by a kind of convolution of the Moebius function $\mu$ with another arithmetic function $\tau$ (which in applications usually is some divisor function; in the above-mentioned case of $k$-free integers, $\tau$ is identically 1 ). A list of special examples will be given in $\S 3$; they improve upon the error terms in some classical asymptotic formulas, assuming the validity of $R H$ throughout.

THEOREM. Let $\tau(k)$ be an arithmetic function satisfying $\tau(k) \geq 0$

$$
D(u):=\sum_{k \leq u} \tau(k)=\sum_{j=1}^{J} \alpha_{j} u^{c_{j}}(\log u)^{b_{j}}+O\left(u^{\lambda}\right) \quad(u \rightarrow \infty)
$$

with $\alpha_{j} \in \mathbf{R}, c_{j} \in \mathbf{R}^{+}, b_{j} \in \mathbf{N}_{0}, \lambda \in \mathbf{R}^{+}$. Furthermore, let $Z(s)$ be a meromorphic function with finitely many poles in the halfplane $\operatorname{Re} s>h$, satisfying (for any $\varepsilon>0)$

$$
Z(\sigma+i t)=O\left(|t|^{\varepsilon}\right)
$$

for $|t| \geq t_{0}, \sigma \geq h+\varepsilon\left(t_{0}, h \in \mathbf{R}^{+}\right)$. Moreover, suppose that

$$
Z(s)=\sum_{k=1}^{\infty} \tau(k) k^{-s}
$$

for $\operatorname{Re} s$ sufficiently large, and

$$
\min \left(c_{j}\right)>h>\lambda .
$$

Received by the editors April 30, 1986 and, in revised form, July 9, 1987.

1980 Mathematics Subject Classification (1985 Revision). Primary 10H25; Secondary 10H05.

Key words and phrases. Arithmetic functions, convolution method, Riemann's Hypothesis. 
Finally, let the arithmetic function $a(n)$ be defined by

$$
a(n)=\sum_{m^{a} k^{b}=n} \mu(m) \tau(k)
$$

where $a, b \in \mathbf{N}, a \lambda<b<2 a h$ and $\mu(m)$ is the Moebius function. Then, assuming the validity of $R H$, we have (for any $\varepsilon>0$ ),

$$
S(x):=\sum_{n \leq x} a(n)=H(x)+O\left(x^{(2 h-\lambda) /(2 a(h-\lambda)+b)+\varepsilon}\right)
$$

where

$$
H(x)=\sum_{\sigma>h / b} \operatorname{Res}\left(\frac{Z(b s)}{\varsigma(a s)} \frac{x^{s}}{s}\right) .
$$

2. Proof of the theorem. We begin by formulating the pivotal auxiliary result which had been given (without a detailed proof) by Montgomery and Vaughan in [5, p. 250].

LEMMA. For a large parameter $y$, define $f_{1}(s)=\sum_{m \leq y} \mu(m) m^{-s}$ and $f_{2}(s)=$ $\varsigma(s)^{-1}-f_{1}(s)$. Then we have for $\sigma \geq \frac{1}{2}+\varepsilon(s=\sigma+i t$ as usual $)$, under the assumption of $R H$,

$$
f_{2}(s)=O\left(y^{1 / 2-\sigma+\varepsilon}\left(|t|^{\varepsilon}+1\right)\right)
$$

for any $\varepsilon>0$.

PROOF. This follows by the argument on p. 315 of [16], in view of the estimate

$$
(\varsigma(s+w))^{-1}=O\left(|\operatorname{Im} s|^{\varepsilon}+|\operatorname{Im} w|^{\varepsilon}+1\right)
$$

which holds for $\operatorname{Re}(s+w) \geq \frac{1}{2}+\varepsilon$ (assuming $R H$ ), for any $\varepsilon>0$ (cf. [16, p. 283]).

To prove the theorem, we first infer by a well-known inversion formula for Dirichlet series (see $[16$, p. 53]) that, for $c$ sufficiently large,

$$
D(u)=(2 \pi i)^{-1} \int_{c-i T}^{c+i T} Z(s) u^{s} s^{-1} d s+O\left(u^{c} T^{-1}\right) .
$$

Shifting the line of integration to $\operatorname{Re} s=h+\varepsilon$ and appealing to (2), we obtain (after choosing $T=T(u)$ sufficiently large)

$$
D(u)=R(u)+O\left(u^{h+\varepsilon}\right), \quad R(u)=\sum_{\sigma>h} \operatorname{Res}\left(Z(s) u^{s} s^{-1}\right)
$$

(with a new $\varepsilon>0$ arbitrarily small). In view of (4), thus (1) may be rewritten as

$$
D(u)=R(u)+O\left(u^{\lambda}\right) .
$$

We now split up (with a parameter $y<x^{1 / a}$ remaining at our disposition)

$$
S(x)=\sum_{\substack{m^{a} k^{b} \leq x \\ m \leq y}} \mu(m) \tau(k)+\sum_{\substack{m^{a} k^{b} \leq x \\ m>y}} \mu(m) \tau(k)=: S_{1}(x)+S_{2}(x) .
$$


The first sum we evaluate by (8), obtaining

$$
\begin{aligned}
S_{1}(x) & =\sum_{m \leq y} \mu(m) D\left(x^{1 / b} m^{-a / b}\right) \\
& =\sum_{m \leq y} \mu(m) R\left(x^{1 / b} m^{-a / b}\right)+O\left(x^{\lambda / b} \sum_{m \leq y} m^{-a \lambda / b}\right) \\
& =\sum_{\sigma>h / b} \operatorname{Res}\left(Z(b s) f_{1}(a s) x^{s} s^{-1}\right)+O\left(x^{\lambda / b} y^{1-a \lambda / b}\right)
\end{aligned}
$$

(since $a \lambda / b<1$ ). To $S_{2}(x)$ we apply again the lemma of [16, p. 53], and get, with $c$ sufficiently large,

$$
S_{2}(x)=(2 \pi i)^{-1} \int_{c-i T}^{c+i T} f_{2}(a s) Z(b s) x^{s} s^{-1} d s+O\left(x^{c} T^{-1}\right)
$$

We now shift the line of integration to $\operatorname{Re} s=\gamma+\varepsilon$, where $\gamma=h / b$ for short; to this end we first infer from (2) and (7) that (with $s=\sigma+i t$ ),

$$
f_{2}(a s) Z(b s)=O\left(y^{1 / 2-a \gamma}\left(|t|^{\varepsilon}+1\right)\right)
$$

choose $T=T(x)$ sufficiently large and obtain (with a new $\varepsilon>0$ ),

$$
S_{2}(x)=\sum_{\sigma>\gamma} \operatorname{Res}\left(Z(b s) f_{2}(a s) x^{s} s^{-1}\right)+O\left(x^{\gamma+\varepsilon} y^{1 / 2-a \gamma}\right) .
$$

Choosing finally $y=x^{1 /(a+b / 2(h-\lambda))}$ and combining the results (10) and (12), we complete the proof of our theorem.

\section{Some applications.}

3.1. Let $a(n)$ denote the number of integer pairs $(p, q)$ for which the least common multiple equals $n$. According to [12, p. 493, Lemma 3.1], (5) is satisfied with $a=2$, $b=1, \tau(k)=d_{3}(k)$ (the number of integer triples $(u, v, w)$ with $u v w=k$ ), hence $Z(s)=\varsigma^{3}(s)$ and $(2)$ is true with $h=\frac{1}{2}$, under $R H$. An asymptotic formula of type (1) was established by G. A. Kolesnik for any $\lambda>43 / 96$ [3]. Hence (assuming $R H)$ our theorem yields

$$
\begin{gathered}
\sum_{n \leq x} a(n)=H(x)+O\left(x^{(1-\lambda) /(3-4 \lambda)+\varepsilon^{\prime}}\right)=H(x)+O\left(x^{53 / 116+\varepsilon}\right), \\
H(x)=A_{1} x(\log x)^{2}+B_{1} x \log x+C_{1} x .
\end{gathered}
$$

This was established in [12], also under $R H$, with the weaker error term $O\left(x^{(2-\lambda) /(5-4 \lambda)+\varepsilon}\right)$ (there also the constants are given explicitly).

3.2. $r$-FREE DIVISORS $(r=2,3)$. An integer is called $r$-free, if it is not divisible by the $r$ th power of any integer $>1$. Let $a_{r}(n)$ denote the number of $r$-free divisors of the positive integer $n(r=2$ or 3$)$. Then condition (5) is met with $a=r$, $b=1, \tau(k)=d(k)$ (the number of divisors). An estimate of type (1) was obtained recently by $\mathrm{H}$. Iwaniec and C. J. Mozzochi for any $\lambda>7 / 22[2]$. With $Z(s)=\varsigma^{2}(s)$, 
$h=1 / 2$, our theorem yields (under $R H$ )

$$
\begin{gathered}
\sum_{n \leq x} a_{2}(n)=H_{2}(x)+O\left(x^{(1-\lambda) /(3-4 \lambda)+\varepsilon^{\prime}}\right)=H_{2}(x)+O\left(x^{15 / 38+\varepsilon}\right) \\
\sum_{n \leq x} a_{3}(n)=H_{3}(x)+O\left(x^{(1-\lambda) /(4-6 \lambda)+\varepsilon^{\prime}}\right)=H_{3}(x)+O\left(x^{15 / 46+\varepsilon}\right) \\
H_{r}(x)=A_{r} x \log x+B_{r} x
\end{gathered}
$$

(For previous weaker versions of these results and the values of the constants, see $[\mathbf{7}, \mathbf{8}, \mathbf{1 4}, \mathbf{1 5}]$.) Note that for $r \geq 4$, the condition $a \lambda / b<1$ cannot be satisfied by a type (1) estimate for the divisor problem, hence we obtain no new result in this case.

3.3. SQUAREFULL NUMBERS. An integer $n$ is called squarefull, if for any prime $p$ which divides $n$, necessarily $p^{2}$ divides $n$. Let $a(n)=1$ if $n$ is squarefull and $a(n)=0$ otherwise. Since $n$ is squarefull if and only if it can be written (uniquely) in the form $n=v^{2} w^{3}$ with $v$ an integer and $w$ squarefree, condition (5) is satisfied with $a=6, b=1, \tau(k)$ the number of integer pairs $(p, q)$ with $p^{3} q^{2}=k$ (cf. [13]). A type (1) formula was established by P. G. Schmidt [9] with $\lambda=27 / 205$. Applying our theorem with $Z(s)=\varsigma(2 s) \varsigma(3 s), h=1 / 4$, we obtain (subject to $R H$ )

$$
\begin{gathered}
\sum_{n \leq x} a(n)=H(x)+O\left(x^{(1-2 \lambda) /(8-24 \lambda)+\varepsilon}\right)=H(x)+O\left(x^{151 / 992+\varepsilon}\right), \\
H(x)=\varsigma\left(\frac{3}{2}\right)(\varsigma(3))^{-1} x^{1 / 2}+\varsigma\left(\frac{2}{3}\right)(\varsigma(2))^{-1} x^{1 / 3}
\end{gathered}
$$

(The previously best known error term, also subject to $R H$, was $O\left(x^{13 / 81+\varepsilon}\right)$; see D. Suryanarayana and R. Sita Rama Chandra Rao [13].)

3.4. THE DIVISOR PROBLEM FOR $(l, r)$-INTEGERS. For fixed integers $1<r<l$, a natural number $d$ is called an $(l, r)$-integer if it can be written in the form $d=v^{l} w$ where $v$ is an integer and $w$ is $r$-free (see [11] and the literature cited there). Let $a(n)$ denote the number of $(l, r)$-integers $d$ which divide $n$. Then, by Lemma 2.8, of [10], condition (5) is satisfied with $a=r, b=1, \tau(k)$ the number of integer triples $(u, v, w)$ with $u^{l} v w=1$. Therefore $Z(s)=\varsigma(l s) \varsigma^{2}(s)$, hence $h=1 / 2$; in view of the condition $a \lambda<b$, we only consider the case $r=2$ or 3 . For $l \geq 4$, a type (1) result is provided by E. Krätzel's Satz 2 in [4] (combined with the recent estimate on the Dirichlet's divisor problem [2]), for any $\lambda>7 / 22$. Our theorem thus implies the asymptotic formulas (subject to $R H$ )

$$
\sum_{n \leq x} a(n)=H_{2, l}(x)+O\left(x^{15 / 38+\varepsilon}\right), \quad(r=2, l \geq 4)
$$

and

$$
\begin{gathered}
\sum_{n \leq x} a(n)=H_{3, l}(x)+O\left(x^{15 / 46+\varepsilon}\right) \quad(r=3, l \geq 4), \\
H_{r, l}(x)=A_{r, l} x \log x+B_{r, l} x .
\end{gathered}
$$

(For earlier weaker results of this kind, see $[10,11]$, where also the constants $A_{r, l}$ and $B_{r, l}$ are given explicitly.) The case $r=2, l=3$ is somewhat exceptional: From Krätzel's formulae (3) and (13) in [4] and the result from [2] we readily derive 
a type (1) estimate for any $\lambda>\lambda_{0}:=\kappa+(1-3 \kappa)(13-18 \kappa) /(53-54 \kappa), \kappa:=7 / 22$, our theorem implies that (subject to $R H$ )

$$
\begin{aligned}
\sum_{n \leq x} a(n) & =H_{2,3}(x)+O\left(x^{\left(1-\lambda_{0}\right) /\left(3-4 \lambda_{0}\right)+\varepsilon}\right) \\
& =H_{2,3}(x)+O\left(x^{265 / 666+\varepsilon}\right) .
\end{aligned}
$$

4. Concluding remark. The argument exhibited in this paper is capable of certain generalizations, in the sense that the classical Riemann zetafunction may be replaced by some other type of zetafunction (generalizing the Moebius function and thus the auxiliary functions $f_{1}(s), f_{2}(s)$ accordingly). A special result in this direction is due to B. Z. Moroz [6].

\section{REFERENCES}

1. A. Axer, Über einige Grenzwertsätze, Sitzungsber. Österr. Akad. Wiss., Abt. II, 120 (1911), 1253-1298.

2. H. Iwaniec and C. J. Mozzochi, On the circle and divisor problems (to appear).

3. G. A. Kolesnik, On the estimation of multiple exponential sums, Recent Progress in Analytic Number Theory (Proc. Durham Sympos. 1979), vol. I, Academic Press, London, 1981, pp. 231-246.

4. E. Krätzel, Teilerprobleme in drei Dimensionen, Math. Nachr. 42 (1969), 275-288.

5. H. L. Montgomery and R. C. Vaughan, The distribution of squarefree numbers, Recent Progress in Analytic Number Theory (Proc. Durham Sympos. 1979), vol. I, Academic Press, London, 1981, pp. 247-256.

6. B. Z. Moroz, On the number of primitive lattice points in plane domains, Monatsh. Math. 99 (1985), 37-42.

7. B. Saffari, Sur le nombre de diviseurs " $r$-free" d'un entier. Cas $r=2$ et $r=3$, C. R. Acad. Sci. Paris 265 (1967), 705-707.

8. __ Sur le nombre de diviseurs "r-libres" d'un entier, et sur les points à coordenées entières dans certains règions du plan, C. R. Acad. Sci. Paris 266 (1968), 601-603.

9. P. G. Schmidt, Zur Anzahl quadratvoller Zahlen in kurzen Intervallen, Acta Arith. 46 (1986), 159-164.

10. M. V. Subbarao and D. Suryanarayana, The divisor problem for $(k, r)$-integers, J. Austral. Math. Soc. 15 (1973), 430-440.

11. D. Suryanarayana, The divisor problem for $(k, r)$-integers. II, J. Reine Angew. Math. 295 (1977), 49-56.

12. D. Suryanarayana and R. Sitaramachandrarao, The number of pairs of integers with 1.c.m. $\leq x$, Arch. Math. (Basel) 21 (1970), 490-497.

13. __, The distribution of square-full integers, Ark. Mat. 11 (1973), 195-201.

14. D. Suryanarayana and V. Siva Rama Prasad, The number of $k$-free divisors of an integer, Acta Arith. 17 (1971), 345-354.

15. _ The number of $k$-free divisors of an integer. II, J. Reine Angew. Math. 276 (1975), 200-205.

16. E. C. Titchmarsh, The theory of the Riemann zeta-function, Oxford, 1951.

INSTITUT FÜr MATHEMATIK, UNIVERSitÄt FÜr BodenKUltur, GREgOR MENDELStrasse 33, A-1180 Vienna, AUStria

Universität Stuttgart, Mathematisches institut A, Postfach 801140 , PFAFFENWALDRING 57, D-7000 STUtTGaRT 80, GeRMANY 